\title{
Inflammatory Pseudotumor of the Anterior Mediastinum Appearing as a Thymic Malignancy: Report of a Case
}

\author{
Go Kanazawa, Yasushi Sakamaki, Tomofumi Oda \\ Department of Chest Surgery, Osaka Police Hospital, Osaka, Japan \\ Email: gaudongogo@gmail.com
}

Received 27 April 2014; revised 20 May 2014; accepted 10 June 2014

Copyright (C) 2014 by authors and Scientific Research Publishing Inc.

This work is licensed under the Creative Commons Attribution International License (CC BY).

http://creativecommons.org/licenses/by/4.0/

(c) (i) Open Access

\begin{abstract}
We treated a patient with inflammatory pseudotumor of the mediastinum that exhibited high uptake of fluorodeoxyglucose on positron emission tomography. A 69-year-old male patient was diagnosed with a mass measuring $70 \mathrm{~mm}$ in diameter in the anterior mediastinum as revealed by computed tomography. The lesion showed strong uptake of 18-fluorine fluorodeoxyglucose with a maximum standardized uptake value of 10.24 on positron emission tomography, which was suggestive of a thymic malignancy. Complete resection of the mass was achieved, and the postoperative pathological examination confirmed an inflammatory pseudotumor of the mediastinum arising in a perithymic lymph node. Despite its rarity, inflammatory pseudotumor should be taken into consideration when diagnosing a mass lesion with characteristics suggestive of thymic neoplasm on fluorodeoxyglucose positron emission tomography-computed tomography.
\end{abstract}

\section{Keywords}

Inflammatory Pseudotumor, Mediastinum, Thymoma, Thymic Carcinoma, 18-Fluorine Fluorodeoxyglucose Positron Emission Tomography

\section{Introduction}

There are few reports on thoracic inflammatory pseudotumor (IPT) mimicking a thymic neoplasm [1] [2]. We herein report a case of IPT of the mediastinum that showed strong enhancement on 18-fluorine fluorodeoxyglucose positron emission tomography (18F-FDG-PET) and was thus suspected to be a thymoma or thymic carcinoma. 


\section{Case Report}

A 69-year-old male patient with stomatitis and anorexia was diagnosed with an anterior mediastinal tumor as revealed by computed tomography (CT) (Figure 1). Following the diagnosis, he underwent FDG-PET. Upon referral to our department, the mass lesion measured $70 \mathrm{~mm}$ in diameter on CT and showed high uptake of ${ }^{18} \mathrm{~F}$ FDG with a maximum standard uptake value $\left(\mathrm{SUV}_{\max }\right)$ of 10.24 on PET (Figure 2). He showed no clinical or laboratory evidence of lymphoma. The lesion was diagnosed as a thymoma or thymic carcinoma based on the CT findings, without biopsy, according to previously described criteria [3]. The patient then underwent complete surgical resection of the mass. With the patient in the supine position, video-assisted thoracoscopic thymectomy was attempted through the left pleural space. However, severe adhesion was encountered between the mass and the left brachiocephalic vein (Figure 3). The adhesion could not be distinguished from tumor invasion. Therefore, the procedure was converted to open resection through a trapdoor incision involving a median sternotomy with extension to an anterior left thoracotomy and a supraclavicular incision. The lesion was completely resected with combined segmental resection of the left brachiocephalic vein. Histologic examination revealed the presence of proliferating spindle cells and oval cells with a background of infiltrating lymphocytes and plasma cells; these findings were compatible with the typical histologic features of IPT (Figure 4). No malignant cells were observed in the resected specimen. The brachiocephalic vein was histologically free from invasion of the pseudotumor. The patient made an uneventful recovery and has been well with no evidence of disease recurrence for 15 months since the surgery.

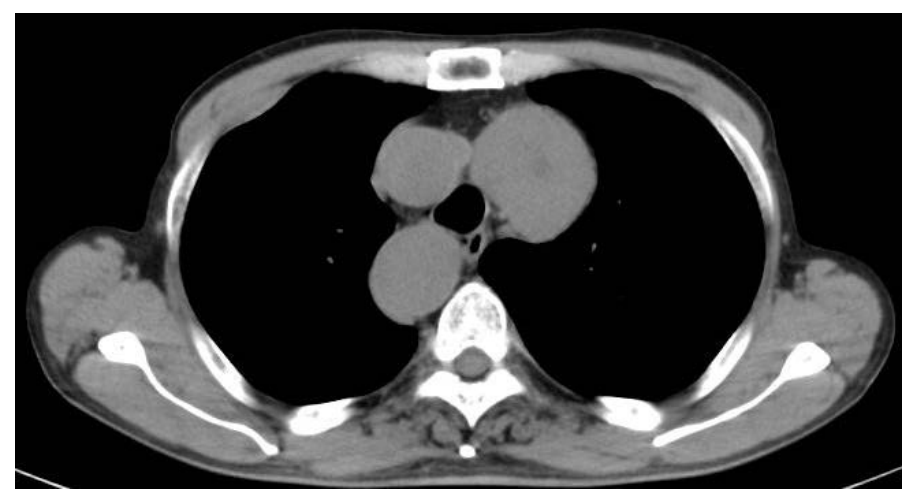

Figure 1. Computed tomography showing a tumor measuring $70 \mathrm{~mm}$ in diameter in the anterior mediastinum and an anomalous right aortic arch.

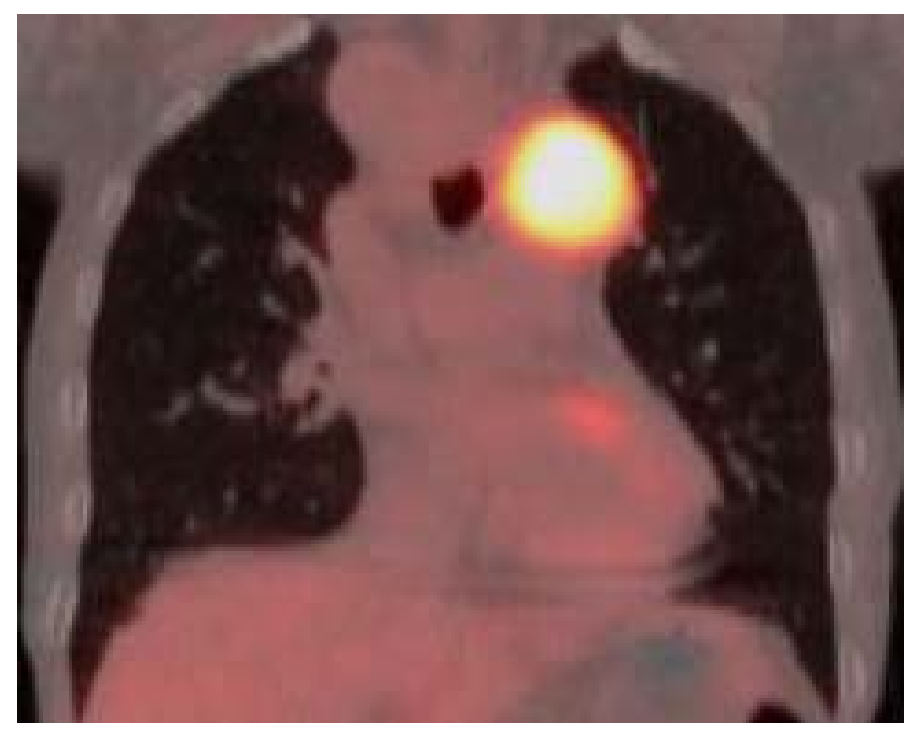

Figure 2. 18-Fluorine fluorodeoxyglucose-positron emission tomography showing abnormally high uptake in the anterior mediastinum. 


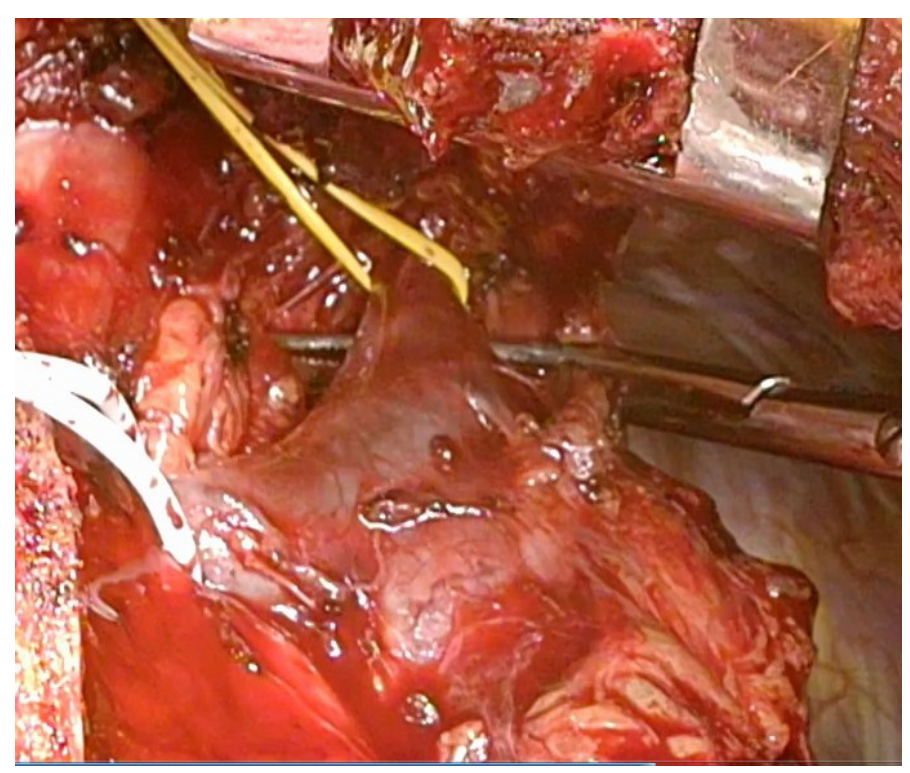

Figure 3. Surgical view showing adhesion between the pseudotumor and the left brachiocephalic vein.

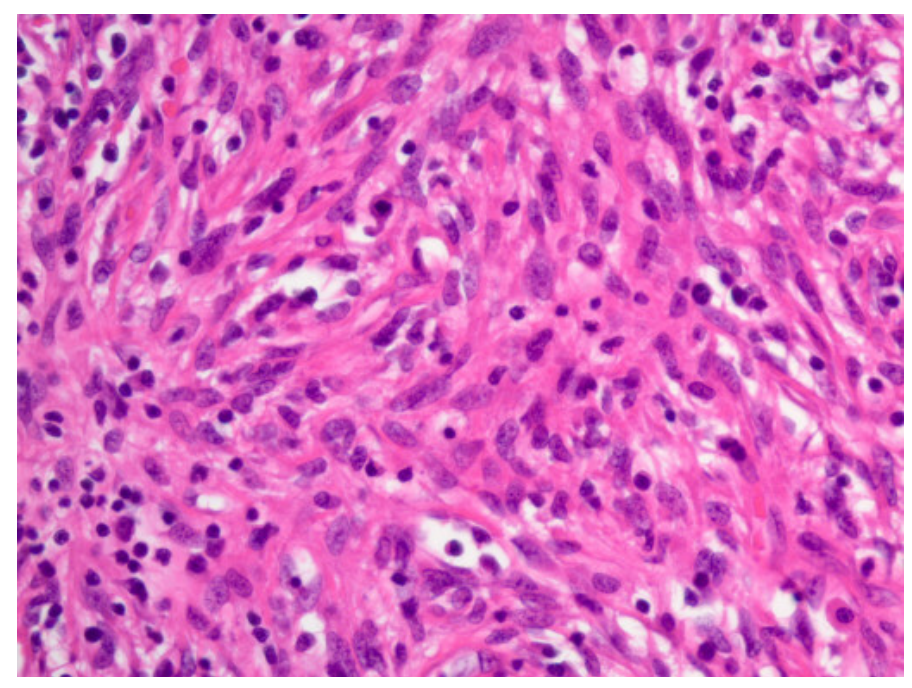

Figure 4. Photomicroscopic findings showing spindle cells with a background of infiltrating plasma cells and lymphocytes.

\section{Discussion}

IPT is a non-neoplastic inflammatory process characterized by a tumor-like appearance as an indolent tumor in most cases [4]. IPT affects both sexes and all races equally, and patient age at presentation ranges from 1 to 73 years [1]. IPT is known to occur in a variety of organs or tissues, including the lung, liver, spleen, urinary tract, and soft tissue; the lung is the most frequent origin [5]. However, we found only a few reports of IPT originating in the anterior mediastinum in a PubMed search of the English-language literature [1] [2].

IPT usually shows oncologic characteristics similar to those of benign tumors, such as a very low incidence of invasion or metastasis [6]. IPT was once believed to be a benign mass lesion containing various proportions of fibroblasts, myofibroblasts, plasma cells, and lymphocytes all recruited in an inflammatory process. Therefore, IPT was termed inflammatory myofibroblastic tumor (IMT), histiocytoma, plasma cell granuloma, or xanthoma depending on the predominant cell in the affected patient [7]. More recently, however, Lawrence et al. [8] reported rearrangement of the anaplastic lymphoma kinase gene in some patients with IMT. Considering these 
findings and a higher likelihood of invasion or metastasis, IMT is currently considered to be a neoplasm and is no longer categorized as IPT [2]. The histologic features commonly seen in IMT were not observed in the present case.

Recent studies reported positive correlations between the malignant grade of thymic epithelial tumors and the SUV $_{\text {max }}$ on FDG-PET [9] [10]. As suggested by these studies, we initially considered the lesion in our case to be thymic carcinoma or thymoma with a high-risk histologic subtype (type B2 or B3) based on the extremely high $\mathrm{SUV}_{\max }$. However, the presence of a high SUV $\mathrm{max}_{\max }$ also depends on various inflammatory cell-mediated processes other than glucose metabolism in tumor cells [10]. Our case suggests that IPT should be included among the differential diagnoses when an abnormally high $\mathrm{SUV}_{\max }$ is observed in a mediastinal mass on FDG-PET.

Surgery is the most reliable modality for both treatment and definitive diagnosis of IPT when appropriate. IPT is usually difficult to diagnose without biopsy, considering its radiologic features. The effectiveness of nonsurgical treatment remains unclear, although the use of antitumor drugs, steroids, and radiotherapy has been reported in patients with unresectable IPT of the anterior mediastinum [2]. Spontaneous regression has been reported in some cases of IPT, but there is currently no method by which to predict spontaneous cure [1]. Whether IPT also has the potential for recurrence or metastasis, as described in reports on IMT, is unclear [2]. Local recurrence of IPT has occasionally been reported [6]; therefore, careful long-term follow-up is needed even after macroscopically complete resection.

\section{Conclusion}

Our case suggests that IPT should be considered in the diagnosis of an anterior mediastinal mass with a high $\mathrm{SUV}_{\max }$ on FDG-PET.

\section{References}

[1] Mountney, J., Suvarna, S.K., Brown, P.W.G. and Thorpe, J.A.C. (1997) Inflammatory Pseudotumor of the Lung Mimicking Thymoma. European Journal of Cardio-Thoracic Surgery, 12, 801-803. http://dx.doi.org/10.1016/S1010-7940(97)00236-4

[2] Chen, C.H., Lin, R.L., Liu, H.C., Chen, C.H., Hung, T.T. and Huang, W.C. (2008) Inflammatory Myofibroblastic Tumor Mimicking Anterior Mediastinal Malignancy. The Annals of Thoracic Surgery, 86, 1362-1364. http://dx.doi.org/10.1016/j.athoracsur.2008.03.031

[3] Tomiyama, N., Müller, N.L., Ellis, S.J., Cleverley, J.R., Okumura, M., Miyoshi, S., Miyoshi, S., Kusumoto, M., et al. (2001) Invasive and Noninvasive Thymoma: Distinctive CT Features. Journal of Computer Assisted Tomography, 25, 388-393. http://dx.doi.org/10.1097/00004728-200105000-00010

[4] Umiker, W.O. and Lverson, L. (1954) Postinflammatory Tumors of the Lung; Report of Four Cases Simulating Xanthoma, Fibroma, or Plasma Cell Tumor. Journal of Thoracic Surgery, 28, 55-63.

[5] Addis, B.J. and Corrin, B. (1990) Inflammatory Pseudorumors. Systemic Pathology. The Lungs. Churchill Livingstone, London, 333-339.

[6] Fabre, D.O., Fadel, E.I., Singhal, S.U., Montpreville, V.I., Mussot, S.A., Mercier, O.L., Mercier, O., Dartevelle, P.G., et al. (2009) Complete Resection of Pulmonary Inflammatory Pseudotumor Has Excellent Long-Term Prognosis. The Journal of Thoracic and Cardiovascular Surgery, 137, 435-440. http://dx.doi.org/10.1016/j.jtcvs.2008.07.009

[7] Imperato, J.P., Folkman, J., Sagerman, R.H. and Cassady, J.R. (1986) Treatment of Plasma Cell Granuloma of the Lung with Radiation Therapy. A Report of Two Cases and a Review of the Literature. Cancer, 57, 2127-2129. http://dx.doi.org/10.1002/1097-0142(19860601)57:11<2127::AID-CNCR2820571107>3.0.CO;2-O

[8] Lawrence, B., Perez-Atayde, A., Hibbard, M.K., Rubin, B., Cin, P. and Pinkus, J. (2000) TPM3-ALK and TPM4-ALK Oncogenes in Inflammatory Myofibroblastic Tumors. The American Journal of Pathology, 157, 377-384. http://dx.doi.org/10.1016/S0002-9440(10)64550-6

[9] Matsumoto, I., Oda, M., Takizawa, M., Waseda, R., Nakajima, K., Kawano, M., Mochizuki, T., Ikeda, H., et al. (2013) Usefulness of Fluorine-18 Fluorodeoxyglucose-Positron Emission Tomography in Management Strategy for Thymic Epithelial Tumors. The Annals of Thoracic Surgery, 95, 305-311. http://dx.doi.org/10.1016/j.athoracsur.2012.09.052

[10] Otsuka, H. (2012) The Utility of FDG-PET in the Diagnosis of Thymic Epithelial Tumors. The Journal of Medical Investigation, 59, 225-234. http://dx.doi.org/10.2152/jmi.59.225 